\title{
Efficacy of Constructing Digital Hybrid Skull-Dentition Images Using an Intraoral Scanner and Cone-Beam Computed Tomography
}

\author{
Joo-Hee Lee $\mathbb{D}^{1},{ }^{1}$ Soo-Hwan Byun, ${ }^{2,3,4}$ Sang-Min Yi, ${ }^{2,3,4}$ In-Young Park, ${ }^{3,4,5}$ \\ Byoung-Eun Yang $\oplus^{2,3,4}$ and Hye-Lim Lee $\mathbb{D}^{1,3,4}$ \\ ${ }^{1}$ Division of Pediatric Dentistry, Hallym University Sacred Heart Hospital, Anyang 14066, Republic of Korea \\ ${ }^{2}$ Division of Oral \& Maxillofacial Surgery, Hallym University Sacred Heart Hospital, Anyang 14066, Republic of Korea \\ ${ }^{3}$ Graduate School of Clinical Dentistry, Hallym University, Chuncheon 24252, Republic of Korea \\ ${ }^{4}$ Institute of Clinical Dentistry, Hallym University, Chuncheon 24252, Republic of Korea \\ ${ }^{5}$ Division of Orthodontics, Hallym University Sacred Heart Hospital, Anyang 14066, Republic of Korea
}

Correspondence should be addressed to Byoung-Eun Yang; face@hallym.or.kr and Hye-Lim Lee; onlylove0210@naver.com

Received 9 November 2021; Revised 27 December 2021; Accepted 29 December 2021; Published 3 March 2022

Academic Editor: Lavinia C. Ardelean

Copyright (C) 2022 Joo-Hee Lee et al. This is an open access article distributed under the Creative Commons Attribution License, which permits unrestricted use, distribution, and reproduction in any medium, provided the original work is properly cited.

Cone-beam computed tomography (CBCT) can distort dentition, and additional imaging is often required. A plaster model to help digitize dental images has been widely used in clinical practice, but there are some inconveniences such as complexity of the process and the risk of damage. The aim of this study was to evaluate the potential for improving dentition imaging with CBCT scans using an intraoral scanner instead of a plaster model. The study used laser model-scanned images of plaster models, imaging from two intraoral scanners, and CBCT images from 20 patients aged 12-18 years. CS 3600 (Carestream Dental, Atlanta, USA) and i700 (Medit, Seoul, Korea) were used as intraoral scanners. The full arch was scanned at once or in three sections using intraoral scanners. The segmented scans were merged to obtain full-arch images. With i700, full-arch images were additionally acquired using its "smart stich" function. The virtual skull-dentition hybrid images obtained from intraoral scanners were superimposed with images obtained using a plaster cast. The difference and distance of coordinate values at each reference point were measured. The average distances from the images obtained with the plaster cast were smaller than $0.39 \mathrm{~mm}$, which is the voxel size of CBCT. Scanning the complete or partial arch using CS 3600 or i700 satisfactorily complemented the CBCT when compared to the plaster model. The virtual skull-dentition hybrid image obtained from intraoral scanners will be clinically useful, especially for patients and surgeons who have difficulty in scanning the complete arch at once.

\section{Introduction}

The utilization of three-dimensional (3D) digital images to create a virtual treatment plan has recently gained popularity in the dental field [1-3]. In particular, cone-beam computed tomography (CBCT) can be used to provide 3D information on a patient's craniomaxillofacial region. Virtual simulations can be used to plan maxillofacial surgery, and computeraided design/manufacturing technologies can be used to create dental wafers and implant surgery guides [4-7]. However,
CBCT images have limitations in providing accurate information for the following two reasons: first, CBCT images are scattered by enamel, restorations, implants, orthodontic devices, etc., resulting in streak artifacts. Second, the X-ray beam, which is a CBCT measurement method, does not always create perfectly uniform images [8-11]. As the teeth are enlarged, distortion of the occlusal surface of the teeth occurs. Therefore, to display accurate information about the dentition in the CBCT image, it is necessary to supplement it with additional dentition imaging. A virtual skull-dentition hybrid image, 


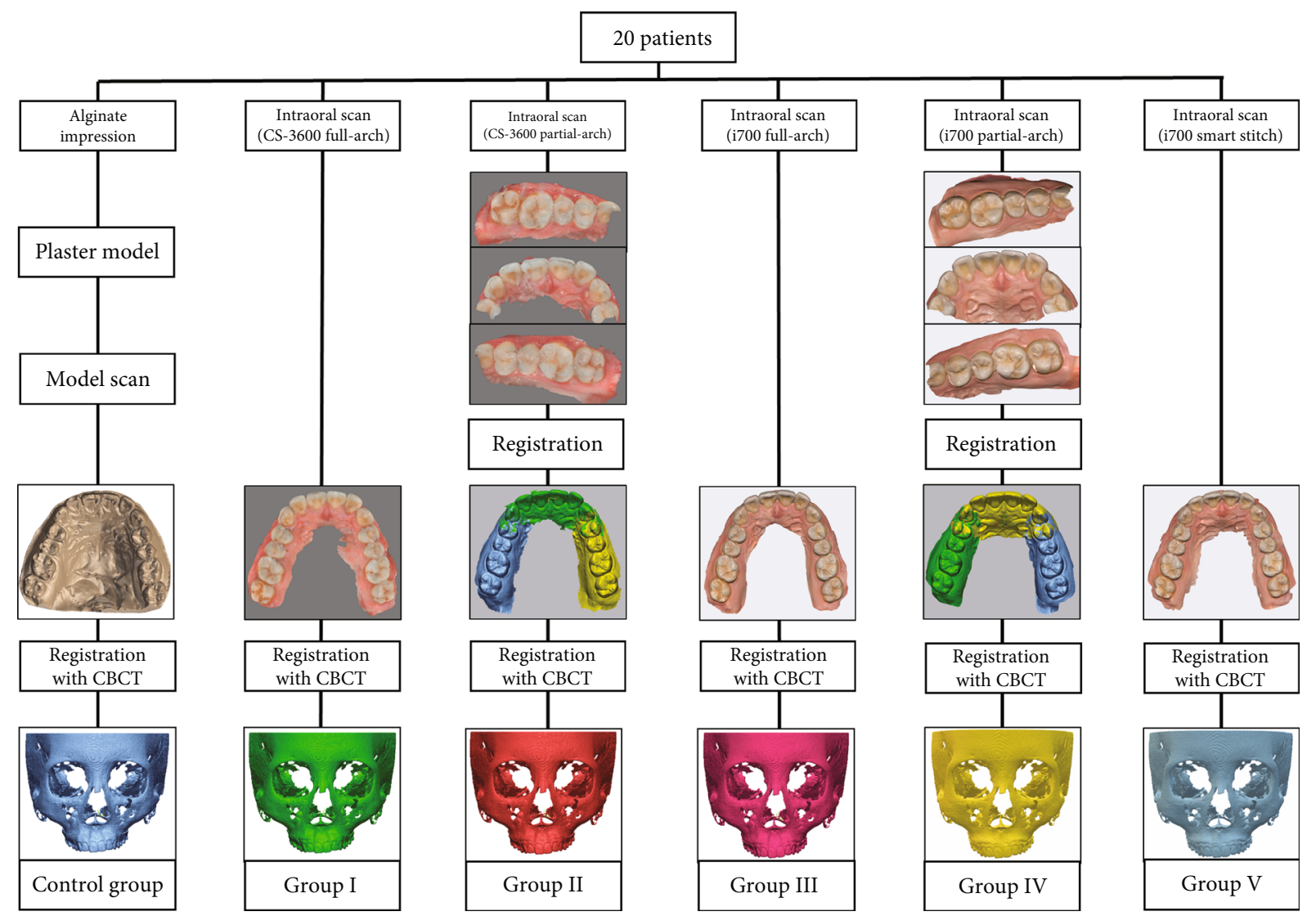

FIGURE 1: Flow charts of the obtaining process of virtual skull-dentition hybrid images of each group.

created by superimposing a dentition image obtained by a model scan of a plaster model with a CBCT image, is a method that has been widely used in clinical practice [12-14].

When compared to laser scanning of plaster casts, utilizing an intraoral scanner (IOS) provides advantages. The IOS is useful because it can obtain a digital model from the patient without having to take a physical impression, and no pouring of plaster is involved. Furthermore, the digital model from the IOS is not susceptible to damage, requires no storage space, and has much fewer time and space limits when collaborating with other departments. In addition, several patients have found intraoral scanning to be more relaxing than traditional impression taking $[15,16]$. Using an IOS, the accuracy of imaging a short span involving one tooth, a quadrant, or sextant is generally comparable to that of the traditional impression method [17]. However, although the accuracy of imaging a long span of the complete arch is improving, it remains controversial $[18,19]$. Imaging of the complete arch varies in accuracy depending on the operator's scan strategy [20]. The entire arch scan data may be obtained by performing segmented scanning, which is more accurate and less affected by the scan method; the segmented images are then integrated to create imaging of a complete arch. The Medit i700 (Medit, Seoul, Korea), which was released in 2021, features a "smart stitch" ability in its software that allows common parts between various scan pieces to be joined automatically. If segmented scanning has a high enough clinical accuracy, it can be employed extensively without being influenced by the operator's scan technique. The goal of this study was to determine whether scanning the entire or partial arch with an IOS can help supplement CBCT scans.

\section{Methods}

The study participants were 20 patients between 12 and 18 years of age (9 males, 11 females), who visited the Dentistry Department of Hallym University Sacred Heart Hospital. The patients' data collection was approved and implemented by the Institutional Review Board (IRB No. 2020-07-005001) of Hallym University Sacred Heart Hospital. The number of study subjects was calculated by using $\mathrm{G}$ * power (ver. 3.0.10, Franz Faul. Universitat, Kiel, Germany) using a significance level of $a=0.05,95 \%$ power, and an effect size of 0.80. Only patients with complete eruption of the first molar were selected; patients with cleft palate, craniofacial syndrome, or metal artifacts such as orthodontic devices or metal restorations were excluded. Plaster cast impression, intraoral scan, and CBCT scans were all performed within a span of two weeks for each patient. A retrospective study was conducted using the maxillary component of each patient's plaster model, IOS digital imaging, and CBCT scan imaging obtained at the time of admission. After obtaining an alginate impression, the surface of the plaster model made with a dental plaster (Rhombstone White, Ryoka Dental, Mie-Ken, Japan) was scanned with a desktop model 


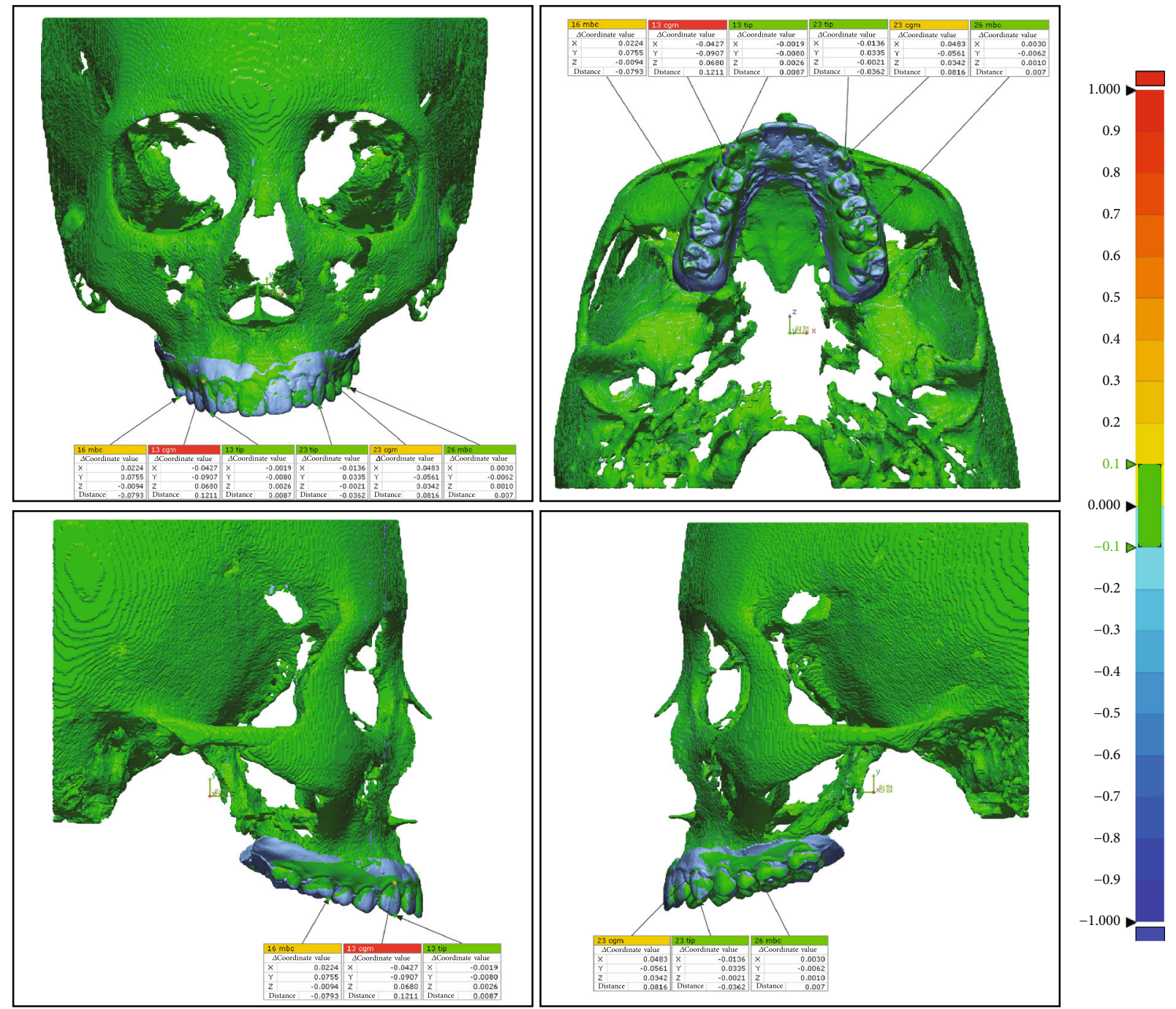

FIgURE 2: Coordinate value difference and distance between reference points after superimposing the control group and group I.

scanner (Freedom UHD, DOF, Inc., Seoul, Korea), and the Surface Tessellation Language (STL) format digital images were acquired. CS 3600 (Carestream Dental, Atlanta, USA) and i700 (Medit, Seoul, Korea) were the intraoral scanners used. For each patient, the same clinician (JH Lee) performed an intraoral scan according to the manufacturer's instructions to acquire digital images in STL format. CBCT was performed using Alphard 3030 (Asahi, Inc., Kyoto, Japan) with a Frankfort plane parallel to the horizontal plane, field of view $200 \times 200 \mathrm{~mm}$, voxel size $0.39 \mathrm{~mm}$, exposure conditions $80 \mathrm{kVP}, 5 \mathrm{~mA}$, and $17 \mathrm{~s}$. CBCT images were converted to Digital Imaging and Communications in Medicine (DICOM) format and reconstructed threedimensionally. Using an IOS, the full arch was scanned simultaneously or divided into sectioned scans. When using the CS 3600, the orthodontic mode was used for scanning the full arch, and the prosthetic mode was used for segmented scanning. The i700 did not have separate modes for full arch and segmented scanning. Additional data were obtained using the i700 "smart stich" function; partial scans were obtained, and the program automatically merged the partial scans to create full-dentition data. For segmented scanning, three divisions were made with the following landmarks: the distal half of the right canine to the distal surface of the right rearmost tooth, the mesial half of the right first premolar to the mesial half of the left first premolar, and the distal half of the left canine to the distal surface of the left rearmost tooth. Subsequently, each divided scanned image was semiautomatically merged based on the overlapping scan images using Geomagic Freeform Plus (3D Systems) to obtain a full-arch dental scan image (STL). Accordingly, six dentition images were obtained from each patient. The CBCT image (DICOM) and each dentition scan image (STL) were transmitted to R2GATE ${ }^{\mathrm{TM}}$ (MegaGen Implant Co., Ltd.). Semiautomatic merging was performed based on the midpoint of the incisal edge of the maxillary left and right central incisors and the mesiobuccal cusp of the maxillary left and right first molars. The final virtual skull-dentition hybrid image was obtained by allowing the anatomical head position to be checked from various directions (Figure 1). 

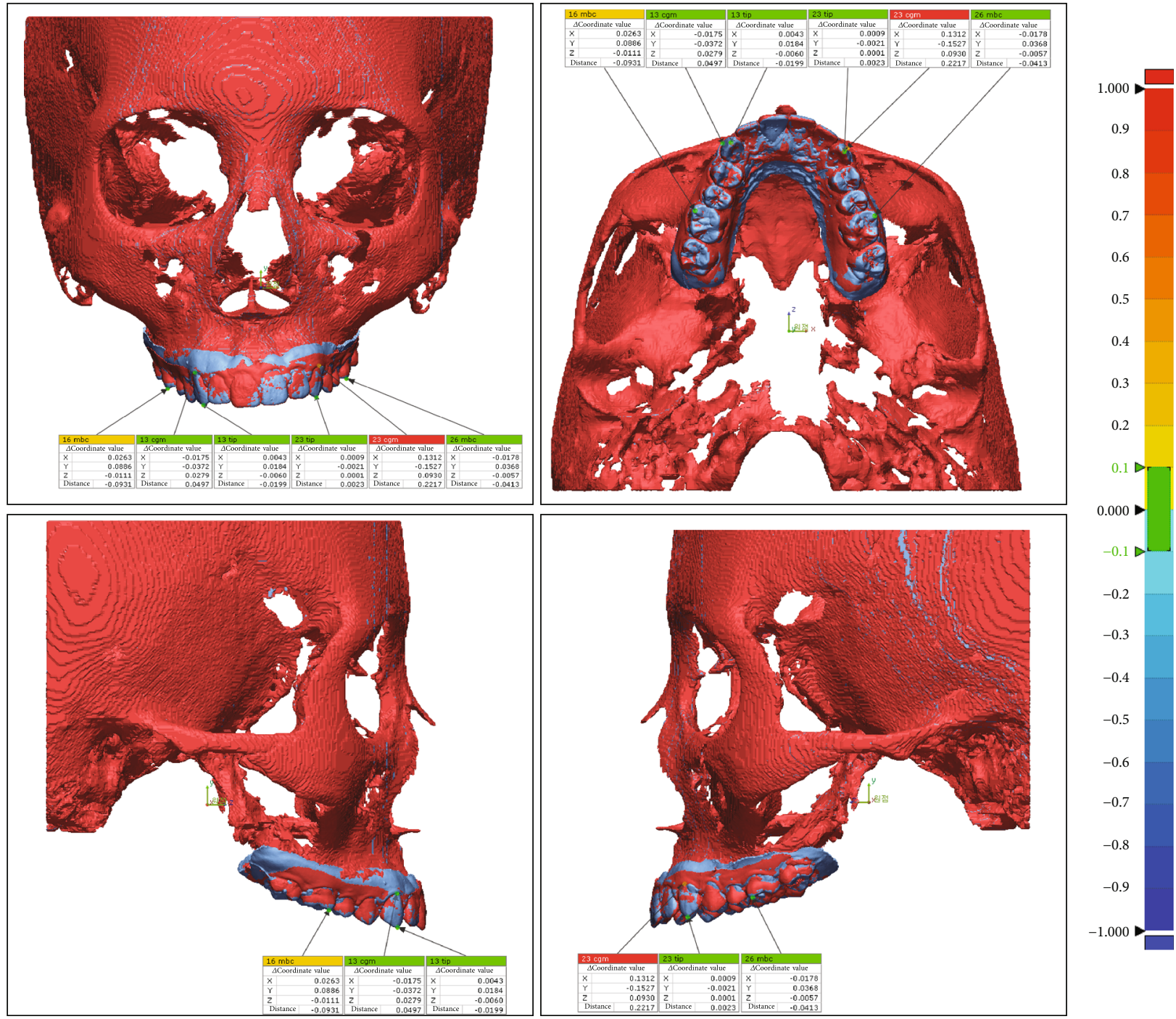

FIGURE 3: Coordinate value difference and distance between reference points after superimposing the control group and group II.

Six virtual skull-dentition hybrid image groups per patient were generated:

(1) Control group: dentition image obtained by a model scan of plaster cast+CBCT scan

(2) Group I: dentition image obtained by scanning the full arch with CS $3600+$ CBCT scan

(3) Group II: dentition image obtained after segmentation scan with CS $3600+$ CBCT scan

(4) Group III: dentition image obtained by scanning the full arch with i700+CBCT scan

(5) Group IV: dentition image obtained after segmentation scan with i700+CBCT scan

(6) Group V: dentition image obtained after segmentation scan with "smart stitch" function of i700+CBCT scan
In the virtual skull-dentition hybrid image of each group, six anatomical reference points were set and evaluated: the cusp of both canines, the lowest point of the gingival margin of both canines, and the mesiobuccal cusp of both first molars. The three-dimensional information of each reference point was expressed as $x, y$, and $z$ coordinate values and entered into a program (Geomagic Freeform Plus, 3D Systems, North Carolina, USA). The $x$-axis showed the left-right direction, the $y$-axis showed the up-down direction, and the $z$-axis showed the relationship in the frontback direction. The difference between the coordinate values at each reference point and the distance between the coordinates were measured by superimposing the digital models of the control group and other groups (Figures 2 and 3). Statistical comparisons were made by performing one-way ANOVA and Tukey post hoc tests on the coordinate values and the distance between the coordinates, respectively. Statistical analysis was performed using the Statistical Package for the Social Sciences (SPSS, version 25.0, IBM). 
TABLE 1: Statistical analysis of the coordinate value difference and distance of each group at the lowest point of the gingival margin of maxillary right canines.

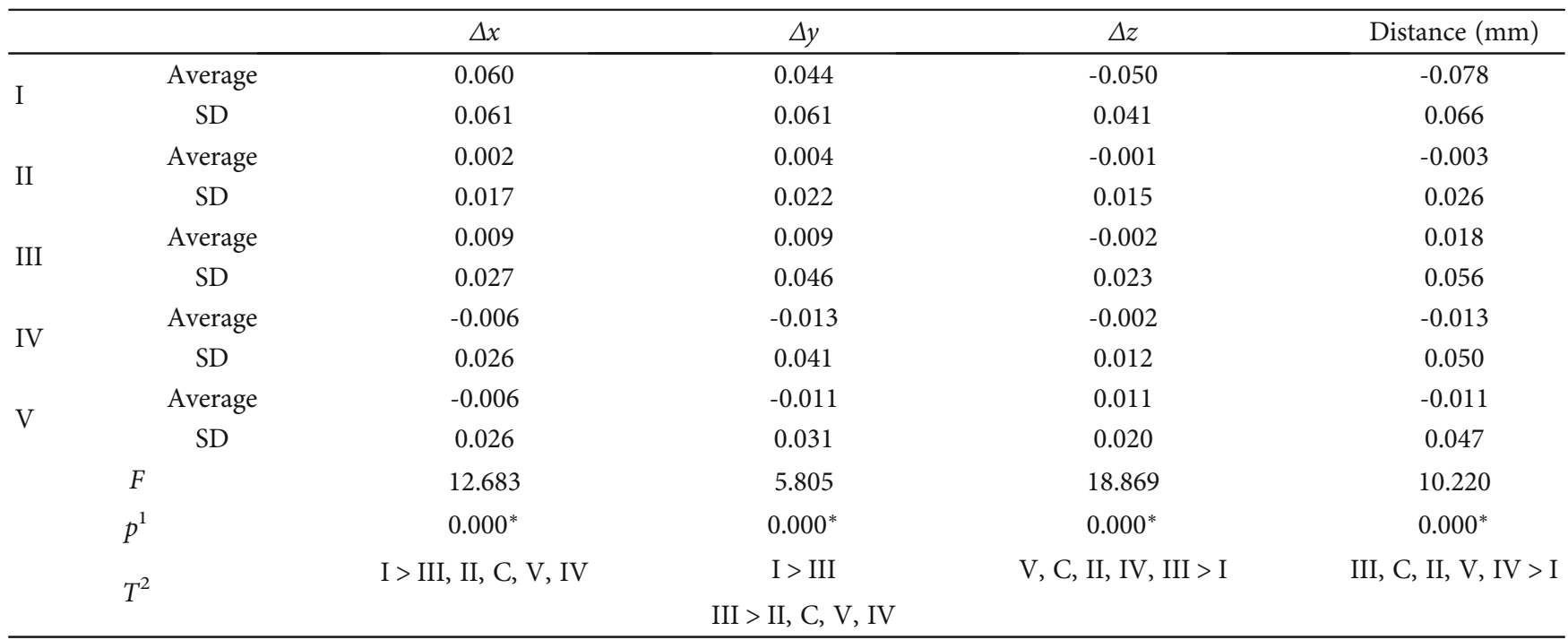

${ }^{1}$ Statistical significances were tested by one-way ANOVA among groups $\left({ }^{*} p<0.05\right) .{ }^{2}$ Adjustment for multiple comparisons: Tukey.

TABLE 2: Statistical analysis of the coordinate value difference and distance of each group at the cusp of maxillary right canines.

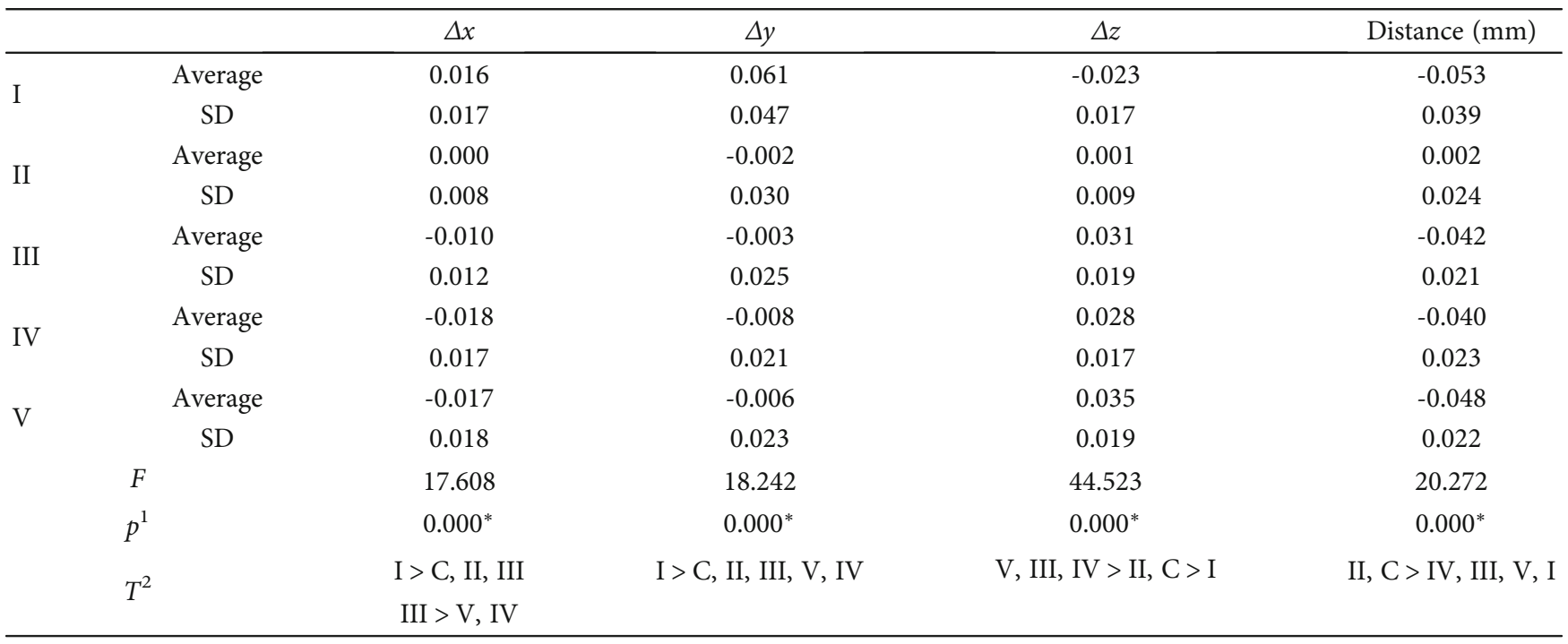

${ }^{1}$ Statistical significances were tested by one-way ANOVA among groups $\left({ }^{*} p<0.05\right) .{ }^{2}$ Adjustment for multiple comparisons: Tukey.

\section{Results}

The mean and standard deviation of each reference point is presented in the tables in this section. The control group is not represented in the table because all values were 0 . Except for the $x$-value of the maxillary left canine cusp, statistical analysis indicated significant differences between the groups. To investigate these discrepancies, a post hoc analysis was performed.

Except for two values (the $x$-value of the cusp of the left maxillary canines and the $z$-value of the mesiobuccal cusp of the left first molars), the mean values of group I were significantly different from those of the control group. On the other hand, except for three values (the distance at the mesiobuccal cusp of the maxillary right first molars, $y$-value of the gingival margin's lowest point of the maxillary left canines, and $y$-value of the cusp of the maxillary left canines), the mean values of group II were not significantly different from those of the control group. In general, groups III, IV, and V produced comparable results. The mean values were highest in group I and lowest in group II, but they were all less than the $0.39 \mathrm{~mm}$ CBCT voxel size (Tables 1-6).

\section{Discussion}

CBCT is an excellent tool to represent a patient's skull, but it can cause distortion of the dentition. The high density of metal restorations, orthodontic appliances, and enamel is the main 
TABLE 3: Statistical analysis of the coordinate value difference and distance of each group at the mesiobuccal cusp of maxillary right first molars.

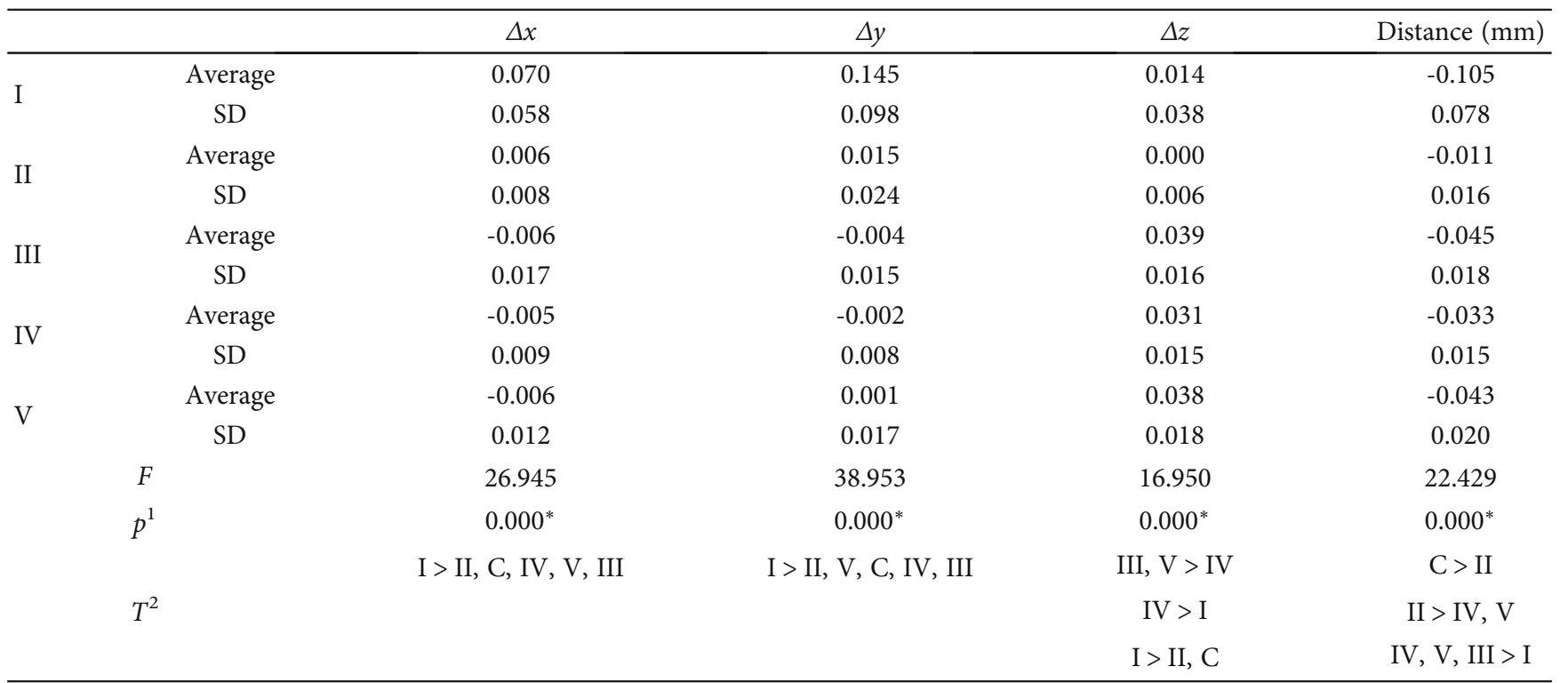

${ }^{1}$ Statistical significances were tested by one-way ANOVA among groups $\left({ }^{*} p<0.05\right) .{ }^{2}$ Adjustment for multiple comparisons: Tukey.

TABLe 4: Statistical analysis of the coordinate value difference and distance of each group at the lowest point of the gingival margin of maxillary left canines.

\begin{tabular}{|c|c|c|c|c|c|}
\hline & & $\Delta x$ & $\Delta y$ & $\Delta z$ & Distance (mm) \\
\hline \multirow{2}{*}{ I } & Average & -0.049 & 0.023 & -0.035 & -0.054 \\
\hline & SD & 0.044 & 0.040 & 0.031 & 0.048 \\
\hline \multirow{2}{*}{ II } & Average & 0.013 & -0.007 & 0.011 & 0.016 \\
\hline & SD & 0.034 & 0.037 & 0.023 & 0.044 \\
\hline \multirow{2}{*}{ III } & Average & 0.000 & -0.002 & 0.005 & -0.008 \\
\hline & SD & 0.017 & 0.017 & 0.015 & 0.028 \\
\hline \multirow{2}{*}{ IV } & Average & 0.004 & -0.008 & 0.001 & -0.011 \\
\hline & $\mathrm{SD}$ & 0.019 & 0.025 & 0.017 & 0.035 \\
\hline \multirow{5}{*}{ V } & Average & -0.002 & 0.004 & -0.002 & 0.004 \\
\hline & SD & 0.030 & 0.030 & 0.027 & 0.050 \\
\hline & $F$ & 12.620 & 3.224 & 11.545 & 8.084 \\
\hline & $p^{1}$ & $0.000^{*}$ & $0.009^{*}$ & $0.000^{*}$ & $0.000^{*}$ \\
\hline & $T^{2}$ & II, IV, C, III, V > I & $\begin{array}{c}\mathrm{I}>\mathrm{V}, \mathrm{C}, \mathrm{III} \\
\mathrm{V}, \mathrm{C}, \mathrm{III}>\mathrm{II}, \mathrm{IV}\end{array}$ & II, III, IV, C, V > I & II, IV, C, III, V > I \\
\hline
\end{tabular}

${ }^{1}$ Statistical significances were tested by one-way ANOVA among groups $\left({ }^{*} p<0.05\right) .{ }^{2}$ Adjustment for multiple comparisons: Tukey.

cause of distortion [8-11]. Additional dentition images from a plaster cast and IOSs were merged into CBCT images to create virtual skull-dentition hybrid images. Errors can occur using IOSs while scanning and processing [21]. Scanning errors are due to a scanning area, the operator's scanning method and skill, and the type of scanner unit [20, 22]. Filter algorithms are to blame for computer processing errors [23, 24].

The control group (a laser model scanning image of a plaster cast) was compared to the five groups acquired by scanning the complete or partial arch with IOSs. Six different reference points on the virtual skull-dentition hybrid images were compared. In terms of coordinates and distance, the segmented scan using the CS 3600 performed best. When employing the full-arch scan method, segmented scan method, and "smart stitch" feature on the i700, comparable results were obtained. All the results of the i700 showed better outcome than the CS 3600 full-arch scan. Although the mean values of group I of the CS 3600 full-arch scan were significantly different from those of the control group, they were all less than the CBCT voxel size of $0.39 \mathrm{~mm}$. The maximum distance was $-0.105 \mathrm{~mm}$ in group I at the mesiobuccal cusp of the maxillary right first molars.

The "smart stitch" function in i700 puts partially scanned images together. There will be more errors when more scan 
TABLE 5: Statistical analysis of the coordinate value difference and distance of each group at the cusp of maxillary left canines.

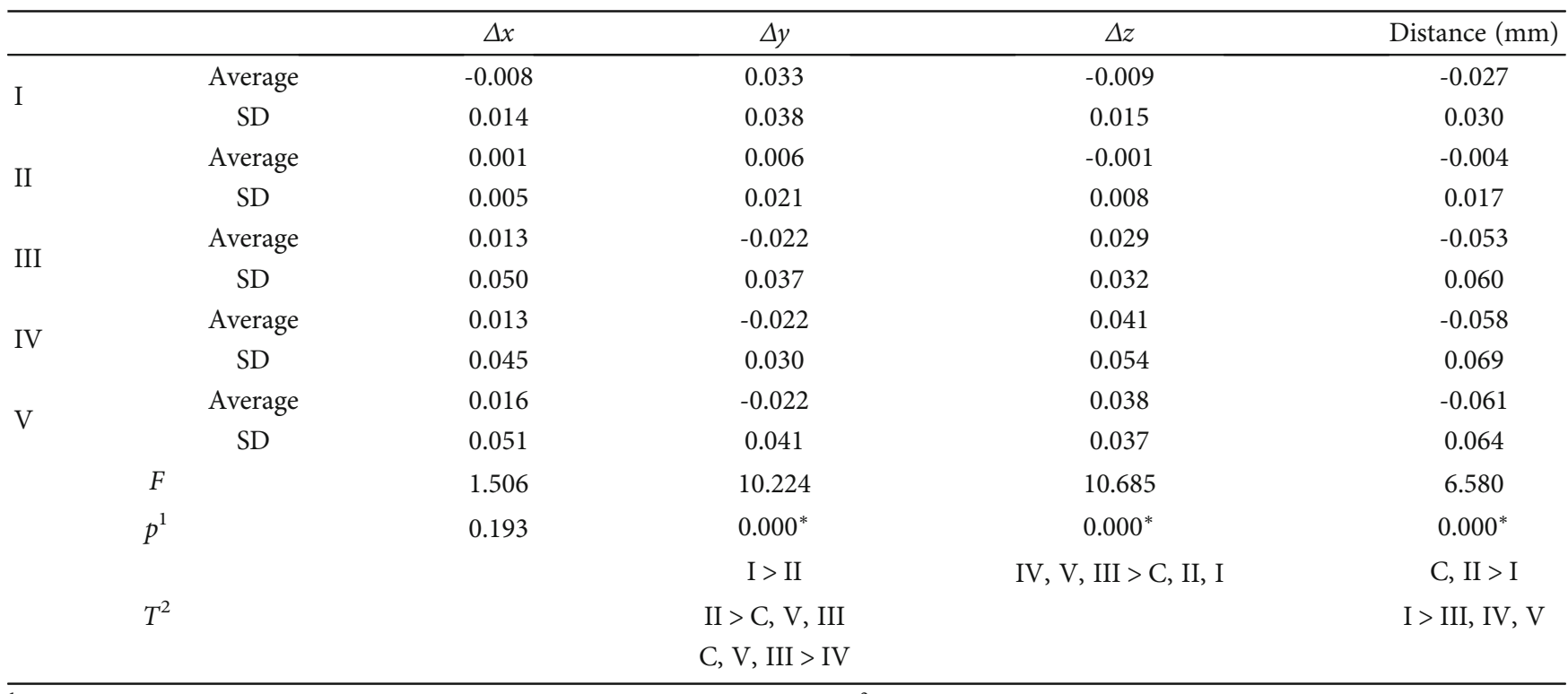

${ }^{1}$ Statistical significances were tested by one-way ANOVA among groups $\left({ }^{*} p<0.05\right) .{ }^{2}$ Adjustment for multiple comparisons: Tukey.

TABLE 6: Statistical analysis of the coordinate value difference and distance of each group at the mesiobuccal cusp of maxillary left first molars.

\begin{tabular}{|c|c|c|c|c|c|}
\hline & & $\Delta x$ & $\Delta y$ & $\Delta z$ & Distance $(\mathrm{mm})$ \\
\hline \multirow{2}{*}{ I } & Average & -0.039 & 0.119 & 0.001 & -0.086 \\
\hline & SD & 0.030 & 0.108 & 0.027 & 0.076 \\
\hline \multirow{2}{*}{ II } & Average & -0.004 & 0.010 & -0.001 & -0.009 \\
\hline & $\mathrm{SD}$ & 0.006 & 0.013 & 0.003 & 0.010 \\
\hline \multirow{2}{*}{ III } & Average & 0.006 & -0.008 & 0.036 & -0.042 \\
\hline & SD & 0.012 & 0.018 & 0.011 & 0.015 \\
\hline \multirow{2}{*}{ IV } & Average & 0.010 & -0.003 & 0.026 & -0.032 \\
\hline & SD & 0.019 & 0.010 & 0.015 & 0.021 \\
\hline \multirow{2}{*}{ V } & Average & 0.004 & -0.004 & 0.036 & -0.041 \\
\hline & SD & 0.023 & 0.015 & 0.018 & 0.026 \\
\hline \multicolumn{2}{|r|}{$F$} & 20.108 & 23.114 & 28.873 & 15.212 \\
\hline \multicolumn{2}{|r|}{$p^{1}$} & $0.000^{*}$ & $0.000^{*}$ & $0.000^{*}$ & $0.000^{*}$ \\
\hline \multicolumn{2}{|r|}{$T^{2}$} & IV, III, V, C, II > I & $\mathrm{I}>\mathrm{II}, \mathrm{C}, \mathrm{IV}, \mathrm{V}, \mathrm{III}$ & III, V, IV > I, C, II & $\begin{array}{c}\text { C, } \text { II }>\text { IV } \\
\text { IV }>\text { V, III }>\text { I }\end{array}$ \\
\hline
\end{tabular}

${ }^{1}$ Statistical significances were tested by one-way ANOVA among groups $\left({ }^{*} p<0.05\right) .{ }^{2}$ Adjustment for multiple comparisons: Tukey.

image stitching is needed [22]. The function does not require more stitching than the full-arch scan method, but it just changes the order of stitching. When comparing the device CS 3600 and the i700, the i700 is lighter in weight, which could explain why the findings are constant regardless of the scanning method. The i700 weighs $245 \mathrm{~g}$ versus $325 \mathrm{~g}$ for the CS 3600 . The i700 scans twice as fast as the i500, Medit's previous scanner, which scans about twice as fast as the CS 3600 .

Baan et al. used a structured light scanner as the gold standard to scan a dried cranium [25]. However, the present study involved real patients, and a control group was created using a virtual skull-dentition hybrid image created using a plaster model. When utilized for orthognathic sur- gery, this type of hybrid image represented the patient's true dentition in a clinically acceptable manner and displayed the desired surgical result without difficulties [26-31]. Despite the likelihood of errors in merging splitscan images, the split-scan method exhibited improved clinical accuracy when CS 3600 was utilized. When aligning the reference point, a study using the same program as this study found a movement error of $139 \mu \mathrm{m}$ and an angle error of 2.52 degrees [32]. Variations in accuracy may occur in the process of fusing the CBCT image and the dental image apart from the accuracy of the dental image. Uechi et al. found a root mean square error of $0.4 \mathrm{~mm}$ in their study [27]. Gateno et al. found that the error ranged from 
0.10 to $0.50 \mathrm{~mm}$ [13], while de Waard et al. found that the error ranged from 0.12 to $0.45 \mathrm{~mm}$ [33].

It is sometimes required to scan only a portion of the dentition rather than the full dentition simultaneously. A fracture line may extend to the teeth in patients who have fractures in the craniofacial region. In this situation, the data needed for surgery can be collected by scanning each section independently, using the fracture line as a reference, and completing a full-arch image. Patients, including children, who have difficulties opening their mouths for extended periods of time, will feel more at ease with a segmented approach. Furthermore, even for operators who are unfamiliar with intraoral scanning, the segmented scanning method will be useful. The extra step of using another program to merge each segmented scan image is time-consuming and difficult. The i700's "smart stitch" feature can make this step obsolete. This is expected to be a beneficial function, as it will merge scan fragments that were split at the time of scanning if there was a common element between them.

One of the limitations of this study was that it only included participants who did not have orthodontic equipment that could distort the CBCT scan. When a patient is receiving orthodontic treatment, a CBCT scan is obtained to assess the progress of the treatment, or maxillofacial surgery is often performed, and a CBCT scan may be of use. Therefore, more research into the effects of orthodontic equipment, such as brackets, is required. Second, the number of patients involved in this study is insufficient; a larger sample size is needed in future studies to support our findings.

\section{Conclusion}

Scanning the complete or partial arch using CS 3600 or i700 satisfactorily complements the CBCT when compared to the plaster model. A partial arch scan with CS 3600 represented the best results, followed by complete arch scan, partial arch scan, and "smart stitch" function with and full arch scan with CS 3600 . This technique shall be widely used clinically for patients and surgeons who find it difficult to scan the complete arch at once, as it can accurately create a virtual skull-dentition hybrid image using an intraoral scanner.

\section{Data Availability}

The data that support the findings of this study are available from the corresponding authors upon reasonable request.

\section{Conflicts of Interest}

The authors declare that they have no conflicts of interest.

\section{Acknowledgments}

This research was supported by Hallym University Research Fund 2021 (HURF-2021-03). Intraoral digital scanners used in the study were purchased with funding from the Medical Device Technology Development program (No. 20006006, title: Development of Artificial Intelligence-based Augmented Reality Surgery System for Oral and Maxillofacial
Surgery by the Ministry of Trade, Industry, and Energy, Republic of Korea).

\section{References}

[1] F. Mangano, J. A. Shibli, and T. Fortin, "Digital dentistry: new materials and techniques," International journal of dentistry, vol. 2016, 2 pages, 2016.

[2] M. Cicciù, L. Fiorillo, C. D’Amico et al., “3D digital impression systems compared with traditional techniques in dentistry: a recent data systematic review," Materials, vol. 13, no. 8, p. $1982,2020$.

[3] M. B. Blatz and J. Conejo, "The current state of chairside digital dentistry and materials," Dental Clinics, vol. 63, no. 2, pp. 175-197, 2019.

[4] F. A. Quereshy, T. A. Savell, and J. M. Palomo, “Applications of cone beam computed tomography in the practice of oral and maxillofacial surgery," Journal of Oral and Maxillofacial Surgery, vol. 66, no. 4, pp. 791-796, 2008.

[5] G. De Vico, F. Ferraris, L. Arcuri, F. Guzzo, and D. Spinelli, "A novel workflow for computer guided implant surgery matching digital dental casts and CBCT scan," ORAL \& implantology, vol. 9, no. 1, p. 33, 2016.

[6] G. Fokas, V. M. Vaughn, W. C. Scarfe, and M. M. Bornstein, "Accuracy of linear measurements on CBCT images related to presurgical implant treatment planning: a systematic review," Clinical Oral Implants Research, vol. 29, no. S16, pp. 393-415, 2018.

[7] W. Derksen, D. Wismeijer, T. Flügge, B. Hassan, and A. Tahmaseb, "The accuracy of computer-guided implant surgery with tooth-supported, digitally designed drill guides based on CBCT and intraoral scanning. A prospective cohort study," Clinical Oral Implants Research, vol. 30, no. 10, pp. 1005-1015, 2019.

[8] A. Katsumata, A. Hirukawa, M. Noujeim et al., "Image artifact in dental cone-beam CT," Oral Surgery, Oral Medicine, Oral Pathology, Oral Radiology, and Endodontology, vol. 101, no. 5, pp. 652-657, 2006.

[9] C. Nardi, C. Borri, F. Regini et al., "Metal and motion artifacts by cone beam computed tomography (CBCT) in dental and maxillofacial study," La Radiologia Medica, vol. 120, no. 7, pp. 618-626, 2015.

[10] B. Al-Rawi, B. Hassan, B. Vandenberge, and R. Jacobs, “Accuracy assessment of three-dimensional surface reconstructions of teeth from cone beam computed tomography scans," Journal of Oral Rehabilitation, vol. 37, no. 5, pp. 352-358, 2010.

[11] M. A. Sanders, C. Hoyjberg, C. B. Chu, V. L. Leggitt, and J. S. Kim, "Common orthodontic appliances cause artifacts that degrade the diagnostic quality of CBCT images," Journal of the California Dental Association, vol. 35, no. 12, pp. 850857, 2007.

[12] C. Mangano, F. Luongo, M. Migliario, C. Mortellaro, and F. G. Mangano, "Combining intraoral scans, cone beam computed tomography and face scans: the virtual patient," Journal of Craniofacial Surgery, vol. 29, no. 8, pp. 2241-2246, 2018.

[13] J. Gateno, J. Xia, J. F. Teichgraeber, and A. Rosen, “A new technique for the creation of a computerized composite skull model," Journal of Oral and Maxillofacial Surgery, vol. 61, no. 2, pp. 222-227, 2003.

[14] X. Z. Lin, T. T. Chen, J. Q. Liu, T. F. Jiang, D. D. Yu, and S. G. Shen, "Point-based superimposition of a digital dental model 
on to a three-dimensional computed tomographic skull: an accuracy study in vitro," British Journal of Oral and Maxillofacial Surgery, vol. 53, no. 1, pp. 28-33, 2015.

[15] J. A. Burzynski, A. R. Firestone, F. M. Beck, H. W. Fields Jr., and T. Deguchi, "Comparison of digital intraoral scanners and alginate impressions: time and patient satisfaction," American Journal of Orthodontics and Dentofacial Orthopedics, vol. 153, no. 4, pp. 534-541, 2018.

[16] M. F. Sfondrini, P. Gandini, M. Malfatto, F. di Corato, F. Trovati, and A. Scribante, "Computerized casts for orthodontic purpose using powder-free intraoral scanners: accuracy, execution time, and patient feedback," BioMed Research International, vol. 2018, 8 pages, 2018.

[17] W. Renne, M. Ludlow, J. Fryml et al., "Evaluation of the accuracy of 7 digital scanners: an in vitro analysis based on 3dimensional comparisons," The Journal of Prosthetic Dentistry, vol. 118, no. 1, pp. 36-42, 2017.

[18] A. Ender, M. Zimmermann, and A. Mehl, "Accuracy of complete-and partial-arch impressions of actual intraoral scanning systems in vitro," International Journal of Computerized Dentistry, vol. 22, no. 1, pp. 11-19, 2019.

[19] P. Phudphong, P. Amornvit, and N. Sirintawat, "Comparison of accuracy of alginate impression and intraoral scanner in model with and without orthodontic brackets," Applied Sciences, vol. 11, no. 13, p. 6037, 2021.

[20] L. Passos, S. Meiga, V. Brigagão, and A. Street, "Impact of different scanning strategies on the accuracy of two current intraoral scanning systems in complete-arch impressions: an in vitro study," International Journal of Computerized Dentistry, vol. 22, no. 4, pp. 307-319, 2019.

[21] P. Amornvit, D. Rokaya, and S. Sanohkan, "Comparison of accuracy of current ten intraoral scanners," BioMed Research International, vol. 2021, 10 pages, 2021.

[22] P. Amornvit, D. Rokaya, C. Peampring, and S. Sanohkan, "Confocal 3D optical intraoral scanners and comparison of image capturing accuracy," Computers, Materials \& Continua, vol. 66, no. 1, pp. 303-314, 2020.

[23] A. Ender and A. Mehl, "Accuracy of complete-arch dental impressions: a new method of measuring trueness and precision," The Journal of Prosthetic Dentistry, vol. 109, no. 2, pp. 121-128, 2013.

[24] Y. Takeuchi, H. Koizumi, M. Furuchi, Y. Sato, C. Ohkubo, and H. Matsumura, "Use of digital impression systems with intraoral scanners for fabricating restorations and fixed dental prostheses," Journal of Oral Science, vol. 60, no. 1, pp. 1-7, 2018.

[25] F. Baan, R. Bruggink, J. Nijsink, T. Maal, and E. Ongkosuwito, "Fusion of intra-oral scans in cone-beam computed tomography scans," Clinical Oral Investigations, vol. 25, no. 1, pp. 7785, 2021.

[26] J.-W. Kim, J.-C. Kim, C.-G. Jeong et al., "The accuracy and stability of the maxillary position after orthognathic surgery using a novel computer-aided surgical simulation system," $B M C$ Oral Health, vol. 19, no. 1, pp. 1-13, 2019.

[27] J. Uechi, M. Okayama, T. Shibata et al., “A novel method for the 3-dimensional simulation of orthognathic surgery by using a multimodal image-fusion technique," American Journal of Orthodontics and Dentofacial Orthopedics, vol. 130, no. 6, pp. 786-798, 2006.

[28] S. S.-P. Hsu, J. Gateno, R. B. Bell et al., "Accuracy of a computer-aided surgical simulation protocol for orthognathic surgery: a prospective multicenter study," Journal of Oral and Maxillofacial Surgery, vol. 71, no. 1, pp. 128-142, 2013.

[29] B. Li, L. Zhang, H. Sun, J. Yuan, S. G. F. Shen, and X. Wang, “A novel method of computer aided orthognathic surgery using individual CAD/CAM templates: a combination of osteotomy and repositioning guides," British Journal of Oral and Maxillofacial Surgery, vol. 51, no. 8, pp. e239-e244, 2013.

[30] E. Nkenke, S. Zachow, M. Benz et al., "Fusion of computed tomography data and optical 3D images of the dentition for streak artefact correction in the simulation of orthognathic surgery," Dentomaxillofacial Radiology, vol. 33, no. 4, pp. 226-232, 2004.

[31] A. Ayoub, M. Rehab, M. O’Neil et al., “A novel approach for planning orthognathic surgery: the integration of dental casts into three-dimensional printed mandibular models," International Journal of Oral and Maxillofacial Surgery, vol. 43, no. 4, pp. 454-459, 2014.

[32] S. O’Toole, C. Osnes, D. Bartlett, and A. Keeling, "Investigation into the accuracy and measurement methods of sequential 3D dental scan alignment," Dental Materials, vol. 35, no. 3, pp. 495-500, 2019.

[33] O. de Waard, F. Baan, L. Verhamme, H. Breuning, A. M. Kuijpers-Jagtman, and T. Maal, "A novel method for fusion of intra-oral scans and cone-beam computed tomography scans for orthognathic surgery planning," Journal of CranioMaxillofacial Surgery, vol. 44, no. 2, pp. 160-166, 2016. 